\title{
IMPLICATIONS OF OBSERVATIONS OF THE SOLAR WIND AND CORONA FOR SOLAR WIND MODELS
}

\author{
W.I. AXFORD AND J.F. MCKENZIE \\ Max-Planck-Institut fuer Aeronomie, \\ 37191 Katlenburg-Lindau, Germany
}

In order to understand the solar wind it is important to note that it has two forms which may be described as "fast" and "slow". The fast wind is clearly associated with coronal holes and has the characteristics of being relatively uniform and stable. The slow wind is quite variable in terms of temperature, composition, magnetic field strength etc., and, in contrast to the fast wind, is not in equilibrium with the corona and transition layer at its base. We contend that the fast wind is the normal, equilibrium wind which must first be accounted for in any theory; whereas the slow wind is associated with transiently-open magnetic field lines emerging from regions of the corona which are magnetically largely closed and not necessarily associated with coronal holes.

The normal (fast) solar wind exhibits microscopic features which indicte that strong wave-particle interactions are prevalent even out to the orbit of Earth. Thus the temperature of the ions is anisotropic with the perpendicular temperature exceeding the parallel temperature. The heavy ion species tend to have the same temperature per atomic mass as the protons and also to have a higher speed, the difference being approximately the Alfvén speed. Within $\sim 1 \mathrm{AU}$ the electrons are cooler than the protons but show strong heat flow effects, including a field-aligned beam of higher energy particles apparently arising from the hottest collisionless regions of the corona. In contrast, the slow wind usually has roughly isotropic ion temperature distributions, and the electrons tend to be hotter than ions. Coulomb collisions appear to play an important role in the slow solar wind, maintaining roughly equal flow speeds and temperatures for all species.

The average magnetic field at the base of coronal holes, based on the field observed at large distances, is unipolar and has a strength of 5-10 Gauss. Such fields may fluctuate as a result of forcing from below but the associated waves have long periods and are inefficient in transmitting energy into interplanetary space, giving little possibility of accounting for the astonishing amount of energy contained in the wind. This amounts to a factor of about 10 increase in the total energy of an electron-proton pair between the corona and the orbit of the earth; the energy flux is about the same as that emitted by the transition layer in magnetically closed regions, as one might expect. Since the only source for this energy lies in the magnetic field, one is driven to conclude that the field at the base of the corona is in fact 
not unipolar and that flare-like eruptions or reconnection events must be prevalent on a small scale.

In fact the magnetic field at chromospheric levels in coronal holes is not uniform but tends to be concentrated at the boundaries of supergranules where it is contained in the "network". The latter occupies only about $5 \%$ of the surface area of a coronal hole and must therefore contain magnetic field with strengths in excess of $100-200$ Gauss at the level where the network is defined by its contrast in brightness with respect to the surrounding chromosphere and photosphere. Since the gas deep within the network is generally heated only by radiative transport and heat conduction from below and at the sides and cools by radiating into space, it must be cooler than the surrounding gas, with a correspondingly smaller scale height and lower density at a given level. As a consequence, the Alfvén speed in the network can be relatively large and reconnection events or "flares" can be easily produced in complex, stressed fields, provided the field is not strictly unipolar. The energy for such "flares" may arise partly from below but is probably mainly the result of fresh magnetic flux being pushed into the network from its sides by the large-scale convection associated with the supergranules. Thus, we may regard the energy of the solar wind as being largely derived from the convective motions of the photosphere, coupling through magnetic field amplification and reconnection into non-thermal forms of energy such as waves, shocks and energetic particles. All of these are capable of providing energy to the corona and solar wind without violating any prejudices arising from classical thermodynamics.

The consequences of small-scale "flare" activity as described here are that there should be a continual production of waves, energetic particles and hot, rapidly-moving plasma, all of which have useful properties as far as creating the solar wind is concerned. In particular, the wave spectrum may contain a large amount of power at relatively high frequencies. This is ideal for producing the observed properties of the heavier ion components as well as providing the radiation pressure required to push the wind as a whole. Energetic particles may have a similar effect in that they can induce waves which couple them to the plasma and also help drive the wind by their own pressure.

We have developed a theory for the high speed solar wind based on a simple dissipation length characterization of wave heating of the coronal plasma close to the Sun. It is shown that solutions with the correct particle and energy fluxes and with a realistic magnetic field, match the requirements on the density at the base of the corona provided the dissipation length is relatively small $(\sim 0.25-0.5$ solar radii). The significant features of these solutions (see Figure 1) are that the acceleration is rapid, with the sonic point at about 2 solar radii, and the maximum proton temperatures are high, namely $8-10 \times 10^{6} \mathrm{~K}$, in agreement with some recent observations. Such 

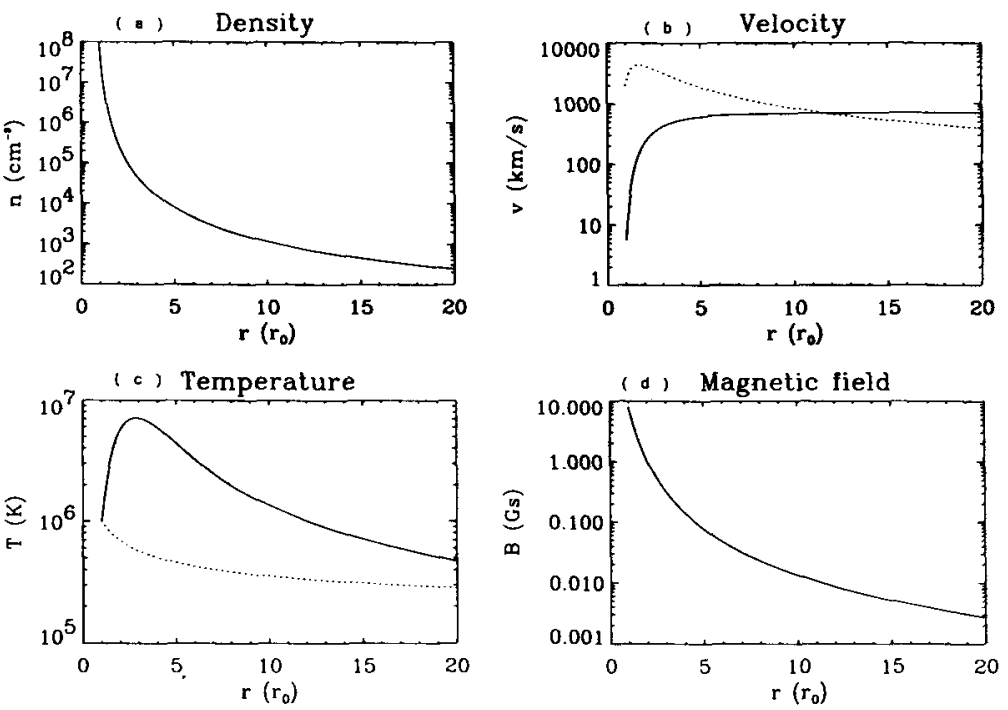

Fig. 1. The density (a), velocity and Alfvén speed (dotted curve)(b), temperature (c) and magnetic field (d) variations with radial distance (in units of solar radii) from the Sun for a high speed solar wind based on a simple dissipation length assumption for hydromagnetic wave heating of the coronal plasma close to the Sun. The significant features, with a dissipation length between 0.25 to 0.5 solar radii, are that rapid acceleration to high speeds, is achieved accompanied by high proton temperatures (full curve in (c)) and low coronal base densities.

efficient dissipation requires any Alfvén waves responsible to have frequencies in the range $0.01 \mathrm{~Hz}-10 \mathrm{kHz}$.

Finally, it is important to note that this perspective on the origin of the high speed solar wind forces us to regard it as originating in strong, complex magnetic field regions covering no more than about $1 \%$ of the solar surface and involving cool (ca. $5000 \mathrm{~K}$ ), relatively low density material. The usuallydefined "base of the corona" is in no sense a boundary condition for the solar wind: this has implications for such aspects as the nature of the plasma (its composition and the state of ionization of the wind) and the energy source in the chromospheric network. 